\title{
Metabolic syndrome: comparison of diagnosis criteria
}

\author{
Síndrome metabólica: comparação de critérios diagnósticos \\ Mônica de Lima Raeder Cavali¹, Maria Arlete Meil Schimith Escrivão², \\ Rosana Sarmento Brasileiro3, José Augusto de Aguiar Carrazedo Taddei ${ }^{4}$
}

\section{Resumo}

Objetivo: Propor um critério para o diagnóstico da síndrome metabólica em adolescentes e verificar a sua concordância com os propostos por Jolliffe e Janssen e pela International Diabetes Federation (IDF).

Método: Estudo transversal com 80 adolescentes obesos de 14 a 19 anos. Foram realizadas avaliações antropométricas (peso, estatura e circunferência da cintura), laboratoriais [triglicérides, high density lipoprotein cholesterol (HDLC), glicemia e insulinemia de jejum] e da pressão arterial. O índice HOMA-IR foi utilizado para caracterizar a resistência insulínica, e a presença de esteatose foi verificada pela ultrassonografia hepática. Análise de concordância entre os três critérios foi feita pela estatística de kappa.

Resultados: Foram encontradas prevalências para síndrome metabólica de $13,75,15$ e $25 \%$, utilizando os critérios da International Diabetes Federation, de Jolliffe e Janssen e da nova proposta, respectivamente. Verificou-se concordância quase perfeita entre Jolliffe e Janssen e a IDF (kappa $=0,94)$ e moderada entre a nova proposta e as duas anteriores (kappa $=0,46$ e 0,41, respectivamente).

Conclusões: A prevalência mais alta da síndrome metabólica foi verificada pelo critério proposto neste estudo, que incluiu a esteatose hepática e a resistência insulínica entre os seus componentes, diagnosticando, desta forma, maior número de adolescentes com risco metabólico.

J Pediatr (Rio J). 2010;86(4):325-330: Resistência insulínica, esteatose hepática, adolescente, síndrome metabólica.

\section{Abstract}

Objective: To propose a new criterion for the diagnosis of metabolic syndrome (MS) in adolescents and to check its consistency with those proposed by Jolliffe and Janssen and by the International Diabetes Federation (IDF).

Method: This is a cross-sectional study of 80 obese adolescents aged 14 to 19 years. Anthropometric (weight, height, and waist circumference) and laboratory (fasting triglycerides, HDLc, glucose, and insulin) parameters, as well as blood pressure were evaluated. The HOMA-IR index was used to characterize insulin resistance, and the presence of steatosis was assessed by hepatic ultrasound. Agreement analyses across the three criteria were made using the kappa coefficient.

Results: The prevalence of MS was 13.5, 15, and 25\% for IDF and Jolliffe and Janssen's criteria and the proposed method, respectively. A nearly perfect agreement between Jolliffe and Janssen's and IDF $(\mathrm{kappa}=0.94)$ criteria and a moderate agreement between the new criteria and the previous two (kappa $=0.46$ and 0.41 , respectively) were observed.

Conclusions: The highest prevalence of MS was observed with the criterion proposed in this study, which included steatosis and insulin resistance as parameters, thus being able to diagnose a larger number of adolescents at metabolic risk.

J Pediatr (Rio J). 2010;86(4):325-330: Insulin resistance, hepatic steatosis, adolescents, metabolic syndrome.

\section{Introdução}

A síndrome metabólica (SM) é definida como uma associação de situações clínicas que inclui hipertensão arterial, dislipidemias, alterações do metabolismo da glicose e obesidade, especialmente abdominal, com depósitos intraabdominais de gordura. A SM representa um agrupamento de fatores de risco para o desenvolvimento das doenças cardiovasculares $(\mathrm{DCV})^{1,2}$.

A prevalência da SM, entre as crianças e os adolescentes, aumentou concomitantemente ao aumento do número de casos de obesidade e suas comorbidades ${ }^{3}$. Análises de dados do National Health and Nutrition Examination Survey (NHANES) III (1988-1992) e do NHANES (1999-2000) constataram aumento da prevalência de SM de 4,2 para 6,4\% na população de adolescentes americanos ${ }^{4}$. Estudo realizado em

1. Mestre, Pediatria, Universidade Estadual de Campinas (UNICAMP), Campinas, SP.

2. Doutora, Pediatria. Chefe, Setor de Obesidade, Disciplina de Nutrologia, Departamento de Pediatria, Universidade Federal de São Paulo (UNIFESP), São Paulo, SP.

3. Doutora, Nutrição, Universidade Federal de São Paulo - Escola Paulista de Medicina (UNIFESP-EPM), São Paulo, SP.

4. Professor associado, Disciplina de Nutrologia, Departamento de Pediatria, UNIFESP, São Paulo, SP.

Este trabalho foi realizado na Disciplina de Nutrologia, Departamento de Pediatria, Universidade Federal de São Paulo (UNIFESP), São Paulo, SP. Apoio financeiro: Este trabalho faz parte de uma pesquisa financiada pela FAPESP (Fundo de Amparo à Pesquisa do Estado de São Paulo), Processo ${ }^{\circ}$ 0754490-8R

Não foram declarados conflitos de interesse associados à publicação deste artigo.

Como citar este artigo: Cavali ML, Escrivão MA, Brasileiro RS, Taddei JA. Metabolic syndrome: comparison of diagnosis criteria. J Pediatr (Rio J). 2010;86(4):325-330

Artigo submetido em 13.01.2010, aceito em 10.03.2010.

doi:10.2223/JPED.2006 
crianças e adolescentes obesos encontrou prevalência da SM de 38,7 e $49,7 \%$ entre aqueles que apresentavam obesidade moderada e grave, respectivamente ${ }^{5}$.

Observa-se ampla variabilidade na prevalência da SM entre os diversos estudos, devido aos diferentes critérios diagnósticos utilizados. Reinehr et al. ${ }^{6}$ compararam a prevalência da SM em crianças e adolescentes utilizando oito diferentes definições. A prevalência variou de 6 a 39\%, e somente $2 \%$ deles preencheram todos os critérios propostos, reforçando a necessidade da padronização de um critério diagnóstico.

Vários critérios para o diagnóstico da SM em crianças e adolescentes já foram propostos ${ }^{5,7-9}$, mas ainda não há consenso na literatura sobre o mais adequado. As constantes mudanças fisiológicas no metabolismo e na composição corporal, que ocorrem nessas faixas etárias, dificultam o estabelecimento de pontos de corte específicos para os parâmetros utilizados nesse diagnóstico. Além disso, faltam estudos de longo seguimento com crianças e adolescentes, associando esses valores com morbidades futuras. O critério para o diagnóstico da SM, na infância e na adolescência, deve conter parâmetros que contemplem as alterações metabólicas de ocorrência mais precoce na criança e no adolescente.

Weiss et al. ${ }^{5}$ observaram que a resistência insulínica (RI) era fator independente para o diagnóstico da SM em crianças e adolescentes com excesso de peso e que a prevalência da SM aumentava com a piora da RI.

O padrão-ouro para a medida da RI é o clamp euglicêmico hiperinsulinêmico, embora ele não seja utilizado na prática clínica ${ }^{10}$. O índice HOMA-IR (homeostasis model assessment - insulin resistance) pode ser usado como método alternativo na avaliação da RI, por apresentar boa correlação com o clamp euglicêmico hiperinsulinêmico ${ }^{11}$.

Chan et al. ${ }^{12}$, a partir de revisão sistemática da literatura, propõem que a doença gordurosa do fígado não alcoólica (DGFNA) seja reconhecida como um componente da SM, por estar envolvida no contexto dos fatores de risco para o desenvolvimento do diabetes melito tipo 2 e da DCV precoce. Musso et al. ${ }^{13}$ verificaram associação positiva entre RI e DGFNA e também sugeriram a inclusão da DGFNA nos critérios para o diagnóstico da SM. D'Ádamo et al. ${ }^{14}$, em estudo transversal com 100 crianças obesas e pré-púberes, encontraram prevalência de esteatose hepática de $52 \%$. As crianças obesas com esteatose, quando comparadas com o grupo-controle de eutróficas e também com aquelas obesas sem esteatose, apresentavam valores de HOMA-IR mais elevados, o que sugere haver associação entre RI e esteatose. Sartorio et al. ${ }^{15}$, avaliando crianças e adolescentes obesos, detectaram esteatose em $44 \%$ deles, e o HOMA-IR também foi maior nos obesos com esteatose, comparados aos obesos sem esteatose.

Apesar das dificuldades para se estabelecer o diagnóstico da SM, cabe ressaltar a importância da identificação de crianças e adolescentes que preencham os requisitos para esse diagnóstico, pois são indivíduos de maior risco metabólico e devem ser adequadamente acompanhados.

O objetivo do presente estudo é propor um novo critério para o diagnóstico da SM em adolescentes obesos e verificar a sua concordância com os propostos por Jolliffe \& Janssen 8 e pela International Diabetes Federation (IDF) ${ }^{9}$.

\section{Métodos}

Trata-se de um estudo transversal, realizado em adolescentes de ambos os sexos, obesos [índice de massa corporal (IMC) > P95], na faixa etária de 14 a 19 anos, matriculados em quatro escolas públicas da mesma região, na cidade de São Paulo (SP). Foram excluídos os adolescentes com doenças agudas ou crônicas, aqueles que estavam em tratamento para perda de peso e as adolescentes grávidas ou em lactação. O estudo foi aprovado pelo Comitê de Ética em Pesquisa da Universidade Federal de São Paulo (UNIFESP), e os dados somente foram coletados após o consentimento de todos os adolescentes e seus responsáveis.

Dos 2.330 adolescentes avaliados, 150 (6,43\%) foram diagnosticados como obesos. Entre estes, 26 (17,33\%) apresentavam os critérios de exclusão citados acima. Dos 124 alunos restantes, $42(33,87 \%)$ recusaram-se a participar do estudo, e dois $(1,61 \%)$ abandonaram o projeto no decorrer da fase de coleta de dados laboratoriais. Chegaram ao final do protocolo, 80 adolescentes obesos (64,5\% da amostra inicial). Quando comparadas as médias dos valores de IMC, estratificados por idade e sexo, da amostra analisada com as médias dos valores de IMC dos 44 adolescentes que não participaram do estudo, não foram encontradas diferenças significantes entre os grupos, evidência de que as perdas não introduziram viés de seleção. Da mesma forma, foram mantidas as proporcionalidades em relação à idade, ao sexo e ao desenvolvimento puberal.

Para a avaliação do peso, foi usada uma balança digital marca Kratos ${ }^{\circledR}$, modelo Linea, e para a estatura, utilizou-se o antropômetro portátil Alturaexata ${ }^{\circledR}$, ambos dispostos em superfície plana e firme. A medida da circunferência da cintura (CC) foi feita no ponto médio entre a última costela e a crista ilíaca, utilizando-se uma fita inextensível. A medida da pressão arterial e o diagnóstico de hipertensão foram feitos de acordo com as recomendações do National High Blood Pressure Education Program Working Group on High Blood Pressure in Children and Adolescents ${ }^{16}$. As amostras de sangue foram coletadas por punção venosa, após 12 horas de jejum, e para as dosagens de glicemia, insulina e perfil lipídico, foram utilizadas técnicas laboratoriais convencionais. Foram considerados os pontos de corte propostos pela American Heart Association (AHA) ${ }^{17}$ para o perfil lipídico. O índice HOMA-IR, utilizado para a classificação de RI, foi obtido multiplicando-se o valor da insulina plasmática de jejum $(\mu \mathrm{U} / \mathrm{mL})$ pelo valor da glicemia de jejum ( $\mathrm{mmol} / \mathrm{L})$ e dividindo-se esse produto por $22,5^{18}$. Valores maiores do que 3,43 foram considerados indicadores de RI ${ }^{19}$.

Ultrassonografia hepática foi realizada pela mesma equipe de radiologistas, com o aparelho modelo LOGIC 400 PROGE. A esteatose foi classificada como mínima, moderada ou grave, segundo Saverymutu et al. ${ }^{20}$.

A nova proposta considerou para o diagnóstico de SM a presença de três ou mais dos seguintes componentes: CC (90 cm para meninos e $80 \mathrm{~cm}$ para meninas) - como su- 
gerido pela IDF $^{21}$ para a população sul-americana, uma vez que ainda não existe uma curva nacional específica e todos os adolescentes apresentavam desenvolvimento puberal $\geq 4$, segundo os critérios de Tanner, hipertensão arterial ${ }^{16}$, hipertrigliceridemia e/ou diminuição de HDLc (high density lipoprotein cholestero/) ${ }^{17}$, RI avaliada pelo HOMA-IR ${ }^{19}$ e presença de esteatose ${ }^{20}$. A Tabela 1 mostra os parâmetros e os respectivos pontos de corte, segundo os diferentes critérios.

A caracterização da amostra foi realizada pela estatística descritiva [média, desvio padrão e intervalo de confiança de 95\% (IC95\%)], e a análise de concordância dos três critérios para o diagnóstico de SM foi feita pela estatística de kappa. Utilizou-se o programa Stata 10.0 para as análises estatísticas ${ }^{22}$.

\section{Resultados}

Do total de 80 adolescentes, $33(41,25 \%)$ eram do sexo masculino. A média de idade foi de 15,96 anos, e a média do IMC, de $32,53 \mathrm{~kg} / \mathrm{m}^{2}$ (Tabela 2). Os adolescentes do sexo masculino apresentaram maior peso $(97,46$ versus $88,81 \mathrm{~kg} ; \mathrm{p}=0,006)$ e maior estatura $(174,05$ versus $164,45 \mathrm{~cm} ; \mathrm{p}=0,000$ ) em relação ao sexo feminino, embora não tenha havido diferença significante no IMC $(p=0,526)$. Não houve diferença significante entre as médias de CC $(p=0,098)$, pressão arterial diastólica $(p=0,336)$, triglicérides $(T G)(p=0,589), \operatorname{HDLc}(p=0,164)$ e $\operatorname{HOMA}(p=0,28)$, quando avaliadas em relação ao sexo, exceto para pressão arterial sistólica $(p=0,040)$. Encontrou-se esteatose em $16,88 \%$ da população estudada (Tabela 2), sendo mais frequente no sexo masculino $(18,18$ versus $15,91 \%)$.
A prevalência da SM variou de acordo com o critério utilizado. O critério da IDF diagnosticou a SM em 13,75\% dos adolescentes; o critério de Jolliffe \& Janssen, em 15\%; e a nova proposta, em $25 \%$ deles.

A concordância no diagnóstico da SM pelos critérios da IDF e de Jolliffe $\&$ Janssen foi quase perfeita (kappa $=0,94$; $p<0,001)$. Os 11 adolescentes que tiveram o diagnóstico de SM pela classificação de Jolliffe \& Janssen também o tiveram pela classificação da IDF, e somente um caso foi diagnosticado apenas pela classificação de Jolliffe \& Janssen. Cabe ressaltar que os 68 casos não diagnosticados pelo IDF também não o foram pelo critério de Jolliffe \& Janssen.

Tabela 2 - Estatística descritiva das características antropométricas, clínicas e laboratoriais dos adolescentes

\begin{tabular}{lcc}
\hline Variável & Média \pm DP & IC95\% \\
\hline Idade (anos) & $15,96 \pm 1,13$ & $15,71-16,21$ \\
Peso $(\mathrm{kg})$ & $92,38 \pm 14,23$ & $89,21-95,55$ \\
Altura $(\mathrm{cm})$ & $168,41 \pm 8,04$ & $166,62-170,20$ \\
IMC $\left(\mathrm{kg} / \mathrm{m}^{2}\right)$ & $32,53 \pm 4,27$ & $31,58-33,48$ \\
CC $(\mathrm{cm})$ & $100,14 \pm 9,66$ & $97,88-102,29$ \\
PAS $(\mathrm{mmHg})$ & $115,23 \pm 11,49$ & $112,68-117,79$ \\
PAD $(\mathrm{mmHg})$ & $70,01 \pm 8,81$ & $68,04-71,97$ \\
TG $(\mathrm{mg} / \mathrm{dL})$ & $86,67 \pm 40,75$ & $77,60-95,74$ \\
HDLc $(\mathrm{mg} / \mathrm{dL})$ & $47,87 \pm 9,25$ & $45,81-49,93$ \\
HOMA-IR & $3,37 \pm 2,19$ & $2,88-3,86$ \\
Esteatose $(\%)$ & 16,88 & - \\
\hline
\end{tabular}

$\mathrm{CC}=$ circunferência da cintura; $\mathrm{DP}=$ desvio padrão; HOMA-IR = homeostasis model assessment - insulin resistance; IC95\% = intervalo de confiança de $95 \%$; IMC = índice de massa corporal; PAD = pressão arterial diastólica; $\mathrm{PAS}=$ pressão arterial sistólica; $\mathrm{TG}=$ triglicérides .

Tabela 1 - Variáveis e pontos de corte segundo as diferentes classificações

\begin{tabular}{|c|c|c|c|}
\hline \multirow[b]{2}{*}{ Variáveis } & \multicolumn{3}{|c|}{ Classificação } \\
\hline & IDF & Jollife \& Janssen 8 & Nova proposta \\
\hline $\mathrm{CC}(\mathrm{cm})$ & & Corte de acordo com idade e sexo & \\
\hline Sexo masculino & $90 \mathrm{~cm}$ & & $90 \mathrm{~cm}$ \\
\hline Sexo feminino & $80 \mathrm{~cm}$ & & $80 \mathrm{~cm}$ \\
\hline Pressão arterial (mm/Hg) & & Corte de acordo com idade e sexo & segundo AAP \\
\hline Sistólica & $\geq 130$ & & \\
\hline Diastólica & $\geq 85$ & & \\
\hline $\mathrm{TG}(\mathrm{mg} / \mathrm{dL})$ & $>150$ & Corte de acordo com idade e sexo & $>150$ segundo $\mathrm{AHA}$ \\
\hline HDLc (mg/dL) & & Corte de acordo com idade e sexo & $<35$ segundo $\mathrm{AHA}$ \\
\hline Sexos masculino e feminino & $<40$ & & \\
\hline Sexo feminino se $>16$ anos & $<50$ & & \\
\hline Glicemia (mg/dL) & $>100$ & $>100$ & - \\
\hline HOMA-IR & - & - & $>3,43$ \\
\hline Ultrassonografia & - & - & presença de esteatose \\
\hline
\end{tabular}

$\mathrm{AAP}=$ American Academy of Pediatrics ${ }^{16} ; \mathrm{AHA}=$ American Heart Association $2003^{17} ; \mathrm{CC}=$ circunferência da cintura; HDLc = high density lipoprotein cholesterol; HOMA-IR = homeostasis model assessment - insulin resistance; IDF = International Diabetes Federation - Zimmet et al. ${ }^{9} ; \mathrm{TG}=$ triglicérides 
A concordância no diagnóstico da SM pelas classificações da IDF e da nova proposta foi moderada (kappa $=0,41$; $\mathrm{p}<0,001$ ) (Tabela 3). Verificou-se que, dos 20 diagnósticos da SM pela nova proposta, oito concordaram, e, em 12, houve diagnóstico apenas pela nova proposta. Os 12 adolescentes diagnosticados pela nova proposta apresentavam RI, e somente um tinha glicemia alterada (103 mg/dL).

Tabela 3 - Número e porcentagem de pacientes, segundo a concordância entre as classificações nova proposta e IDF

\begin{tabular}{lccc}
\hline & \multicolumn{2}{c}{ IDF } & \\
\cline { 2 - 3 } Nova proposta & $\begin{array}{c}\text { Sem SM } \\
\mathbf{n}(\%)\end{array}$ & $\begin{array}{c}\text { Com SM } \\
\mathbf{n}(\%)\end{array}$ & $\begin{array}{c}\text { Total } \\
\mathbf{n}(\%)\end{array}$ \\
\hline Sem SM & $57(95)$ & $3(5)$ & $60(100)$ \\
Com SM & $12(60)$ & $8(40)$ & $20(100)$ \\
Total & $69(86,25)$ & $11(13,75)$ & $80(100)$ \\
\hline
\end{tabular}

IDF = International Diabetes Federation - Zimmet et al. ${ }^{9}$; kappa $=0,41(p<0,001) ; S M=$ síndrome metabólica.

Do mesmo modo, ao serem analisados os resultados obtidos pelas classificações de Jolliffe \& Janssen e da nova proposta, houve concordância moderada no estabelecimento do diagnóstico ( $k a p p a=0,46 ; p<0,001$ ) (Tabela 4). Enquanto a classificação de Jolliffe $\&$ Janssen estabeleceu isoladamente o diagnóstico em três adolescentes, a nova proposta o fez em 11 . Os 11 casos diagnosticados pela nova proposta apresentavam RI. Os três adolescentes, que tiveram diagnóstico de SM pelas classificações da IDF e de Jolliffe \& Janssen, e não pela nova proposta, foram os mesmos.

Entre todos os adolescentes com diagnóstico de RI, 70\% apresentavam glicemia de jejum normal.

Tabela 4 - Número e porcentagem de pacientes, segundo a concordância entre as classificações nova proposta e Jollife \& Janssen

\begin{tabular}{lccc}
\hline & \multicolumn{2}{c}{ Jollife \& Janssen } & \\
\cline { 2 - 3 } Nova proposta & $\begin{array}{c}\text { Sem SM } \\
\mathbf{n}(\%)\end{array}$ & $\begin{array}{c}\text { Com SM } \\
\mathbf{n}(\%)\end{array}$ & $\begin{array}{c}\text { Total } \\
\mathbf{n}(\%)\end{array}$ \\
\hline Sem SM & $57(95)$ & $3(5)$ & $60(100)$ \\
Com SM & $11(55)$ & $9(45)$ & $20(100)$ \\
Total & $68(85)$ & $12(15)$ & $80(100)$ \\
\hline
\end{tabular}

Jollife \& Janssen ${ }^{8}$ kappa = 0,46 $(p<0,001) ; S M=$ síndrome metabólica .
Quando foram analisados os resultados do ultrassom hepático, verificou-se esteatose em 13 adolescentes, sendo que sete pertenciam ao grupo com diagnóstico de SM. Importante salientar que, destes sete, cinco tiveram o diagnóstico da SM somente pela nova proposta, e os outros dois, pelas três classificações. Houve diferença significante entre as médias do HOMA-IR quando se considerou a presença ou ausência de esteatose $(4,68$ versus 3,$05 ; p=0,001)$. A média do HOMA-IR foi significantemente mais elevada na presença de esteatose.

\section{Discussão}

Observa-se, na literatura, grande variação na prevalência da SM em crianças e adolescentes, devido aos diferentes critérios diagnósticos utilizados $6,23,24$. A maioria deles deriva dos critérios usados para o adulto, e, dessa forma, anormalidades que ocorrem com frequências mais baixas na infância e na adolescência, como é o caso da glicemia alterada, podem estar incluídas entre os requisitos para esse diagnóstico.

No presente estudo, a prevalência da SM variou de acordo com o critério utilizado, sendo de $13,75 \%$ para o da IDF, $15 \%$ para o de Jolliffe $\&$ Janssen e $25 \%$ para o da nova proposta. Outros autores, que compararam a prevalência de SM usando diferentes critérios, também encontraram variações entre os valores 23,24 .

A grande concordância na prevalência da SM entre os critérios de Jolliffe \& Janssen e da IDF pode ser explicada pela utilização dos mesmos parâmetros, embora com pontos de corte diferentes. A nova proposta apresentou concordância moderada com os critérios de Jolliffe $\&$ Janssen e da IDF, mas fez diagnóstico de SM em maior percentual de adolescentes, o que pode ser explicado pela substituição da glicemia pela RI ( 30 adolescentes com RI, sendo somente nove com glicemia $\geq 100 \mathrm{mg} / \mathrm{dL}$ ). Weiss et al. ${ }^{5}$ chamam a atenção para o fato de a glicemia de jejum alterar-se tardiamente na evolução da SM nas crianças e nos adolescentes. Neste estudo, foi utilizado o ponto de corte para RI sugerido por Cuartero ${ }^{19}$, devido à RI fisiológica presente na adolescência. Invitti et al. ${ }^{25}$ consideraram a RI como um dos parâmetros para a definição da SM em crianças obesas e encontraram prevalência dessa síndrome em $23,3 \%$, valor muito próximo ao encontrado pela nova proposta (25\%). Ferreira et al. ${ }^{26}$ realizaram um estudo transversal com 52 crianças obesas de 7 a 10 anos e encontraram SM em $10 \%$ dos meninos e $25 \%$ das meninas. Também observaram que, quanto maior era o valor do HOMA-IR, maior era o número de fatores de risco cardiovascular. Do mesmo modo, Caranti et al. 27 , em estudo comparativo com adolescentes obesos brasileiros e italianos, verificaram diferenças entre as duas populações quanto à prevalência da SM, sendo de $23,6 \%$ para os brasileiros e $16,5 \%$ para os italianos. O HOMA-IR foi o parâmetro que apresentou maior frequência de alterações nos dois grupos e era mais elevado entre os adolescentes brasileiros.

Os pontos de corte para a hipertensão arterial foram diferentes entre os três critérios usados neste estudo. A classificação da IDF, com pontos de corte mais altos e sem estratificação para idade, sexo e altura, fez o diagnóstico 
de hipertensão arterial em menor número de adolescentes ( $8 \%)$, em relação ao critério de Jolliffe \& Janssen $(13,75 \%)$ e da nova proposta $(23,75 \%)$.

Encontrou-se maior prevalência de alterações para o HDLc quando utilizado o critério de Jolliffe \& Janssen, com pontos de corte mais elevados em relação às outras duas classificações. A nova proposta baseou-se nos valores preconizados pela $\mathrm{AHA}^{17}$, considerados de risco para a DCV. Os TG estavam alterados em sete adolescentes, com somente uma discordância entre as três classificações. Sinaiko et al. ${ }^{28,}$ em estudo longitudinal com adolescentes de 11 a 14 anos, encontraram no grupo com resistência à insulina, caracterizada pelo clamp euglicêmico hiperinsulinêmico, valores de TG mais elevados e de HDLc mais baixos em relação aos encontrados neste estudo.

Alguns autores consideram a presença da esteatose como a manifestação hepática da RI ${ }^{13,15}$. No estudo de Schwimmer et al. ${ }^{29}$, as crianças e os adolescentes que apresentavam DGFNA tinham valores absolutos mais altos para marcadores de risco cardiovascular, além de maior frequência da SM. Vitola et al. ${ }^{30}$ verificaram diminuição de TG intra-hepáticos, pela ressonância magnética, assim como melhora da sensibilidade à insulina em adolescentes obesos após programa de perda de peso. No presente estudo, a esteatose hepática foi detectada pela ultrassonografia, que é um método de fácil realização, mas, segundo Saadeh et al. ${ }^{31}$, ele detecta alterações quando já ocorreu comprometimento do parênquima hepático maior do que $33 \%$, revelado pela biópsia. A esteatose foi encontrada em 13 adolescentes, e em sete ela estava associada à RI, sugerindo que a esteatose deva ser investigada na presença de RI. A sua inclusão no diagnóstico da SM talvez permita identificar indivíduos com maior risco metabólico.

\section{Conclusões}

A prevalência da SM variou de acordo com o critério diagnóstico utilizado. A prevalência mais alta foi verificada pelo critério proposto neste estudo, que incluiu a RI e a esteatose hepática entre os seus parâmetros, diagnosticando, desta forma, maior número de adolescentes com risco metabólico. A nova proposta fez mais diagnósticos de SM em relação aos outros dois critérios utilizados pelo fato de usar a RI, e não a glicemia, como parâmetro de alteração do metabolismo da glicose. A glicemia costuma alterar-se tardiamente na evolução da SM nas crianças e nos adolescentes.

Cabe ressaltar a importância da identificação de crianças e adolescentes que preencham os requisitos para o diagnóstico da SM, pois são indivíduos de maior risco metabólico e devem ser adequadamente acompanhados. A persistência dessas alterações favorecerá o desenvolvimento do diabetes melito tipo 2 e a ocorrência das DCV na fase adulta.

\section{Referências}

1. Eckel RH, Grundy SM, Zimmet PZ. The metabolic syndrome. Lancet. 2005;365:1415-28.

2. Franks PW, Hanson RL, Knowler WC, Sievers ML, Bennett PH, Looker HC. Childhood obesity, other cardiovascular risk factors, and premature death. N Engl J Med. 2010;362:485-93.
3. Ogden $\mathrm{CL}$, Carroll MD, Curtin LR, McDowell MA, Tabak CJ, Flegal KM. Prevalence of overweight and obesity in the United States, 1999-2004. JAMA. 2006;295:1549-55.

4. Duncan GE, Li SM, Zhou XH. Prevalence and trends of a metabolic syndrome phenotype among U.S. adolescents,1999-2000. Diabetes Care. 2004;27:2438-43.

5. Weiss R, Dziura J, Burgert TS, Tamborlane WV, Taksali SE, Yeckel $\mathrm{CW}$, et al. Obesity and the metabolic syndrome in children and adolescents. N Engl J Med. 2004;350:2362-74.

6. Reinehr T, de Souza G, Toschke AM, Andler W. Comparison of metabolic syndrome prevalence using eight different definitions: a critical approach. Arch Dis Child. 2007;92:1067-72.

7. Cook S, Weitzman M, Auinger P, Nguyen M, Dietz WH. Prevalence of a metabolic syndrome phenotype in adolescents: findings from the third National Health and Nutrition Examination Survey, 19881994. Arch Pediatr Adolesc Med. 2003;157:821-7.

8. Jolliffe $\mathrm{CJ}$, Janssen I. Development of age-specific adolescent metabolic syndrome criteria that are linked to the Adult Treatment Panel III and International Diabetes Federation criteria. J Am Coll Cardiol. 2007;49:891-8.

9. Zimmet P, Alberti KG, Kaufman F, Tajima N, Silink M, Arslanian $\mathrm{S}$, et al. The metabolic syndrome in children and adolescents: the IDF consensus. Diabetes Voice. 2007;52(4):29-32.

10. Consensus Development Conference on Insulin Resistance. 5-6 November 1997. American Diabetes Association. Diabetes Care.1998;21:310-4.

11. Wallace TM, Levy JC, Matthews DR. Use and abuse of HOMA modeling. Diabetes Care. 2004;27:1487-95.

12. Chan HL, de Silva HJ, Leung NW, Lim SG, Farrell GC; Asia-Pacific Working Party on NAFLD. How should we manage patients with non-alcoholic fatty liver disease in 2007? J Gastrenterol Hepatol. 2007;22:801-8.

13. Musso G, Gambino R, Bo S, Uberti B, Biroli G, Pagano G, et al. Should nonalcoholic fatty liver disease be included in the definition of metabolic syndrome? A cross-sectional comparison with Adult Treatment Panel III criteria in nonobese nondiabetic subjects. Diabetes Care. 2008;31:562-8.

14. D'Adamo E, Santoro N, Caprio S. Metabolic syndrome in pediatrics: old concepts revised, new concepts discussed. Endocrinol Metab Clin North Am. 2009;38:549-63.

15. Sartorio A, Del Col A, Agosti F, Mazzilli G, Bellentani S, Tiribelli $C$, et al. Predictors of non-alcoholic fatty liver disease in obese children. Eur J Clin Nutr. 2007;61:877-83.

16. National High Blood Pressure Education Program Working Group on High Blood Pressure in Children and Adolescents. The fourth report on the diagnosis, evaluation, and treatment of high blood pressure in children and adolescents. Pediatrics. 2004;114:555-76.

17. Kavey RE, Daniels SR, Lauer RM, Atkins DL, Hayman LL, Taubert $K$. American Heart Association guidelines for primary prevention of atherosclerotic cardiovascular disease beginning in childhood. Circulation. 2003;107:1562-6.

18. Matthews DR, Hosker JP, Rudenski AS, Naylor BA, Treacher DF, Turner RC. Homeostasis model assessment: insulin resistance and beta-cell function from fasting plasma glucose and insulin concentrations in man. Diabetologia. 1985;28:412-9.

19. Cuartero BG, Lacalle CG, Lobo CJ, Vergaz AG, Rey CC, Villar MJ, et al. Índice de HOMA y QUICKI, Insulina y peptide $C$ en niños sanos. Puntos de corte de riesgo cardiovascular. An Pediatr (Barc). 2007;66:481-9.

20. Saverymuttu SH, Joseph AE, Maxwell JD. Ultrasound scanning in the detection of hepatic fibrosis and steatosis. Br Med J (Clin Res Ed). $1986 ; 292: 13-5$

21. Alberti KG, Zimmet P, Shaw J; IDF Epidemiology Task Force Consensus Group. The metabolic syndrome-a new world-wide definition. Lancet. 2005;366:1059-62.

22. Stata Statistical Software. Release 10.0. College Station, TX: Stata Corporation; 2003 
23. Golley RK, Magarey AM, Steinbeck KS, Baur LA, Daniels LA. Comparison of metabolic syndrome prevalence using six different definitions in overweight pre-pubertal children enrolled in a weight management study. Int J Obes (Lond). 2006;30:853-60.

24. Sartorio A, Agosti F, De Col A, Mornati D, Francescato MP, Lazzer $\mathrm{S}$. Prevalence of the metabolic syndrome in Caucasian obese children and adolescents: comparison between three different definition criteria. Diabetes Res Clin Pract. 2007;77:341-2.

25. Invitti C, Maffeis C, Gilardini L, Pontiggia B, Mazzilli G, Girola A, et al. Metabolic syndrome in obese Caucasian children: prevalence using WHO-derived criteria and association with nontraditional cardiovascular risk factors. Int J Obes (Lond). 2006;30:627-33.

26. Ferreira AP, Oliveira CE, França NM. Metabolic syndrome and risk factors for cardiovascular disease in obese children: the relationship with insulin resistance (HOMA-IR). J Pediatr (Rio J). 2007;83:21-6.

27. Caranti DA, Lazzer S, Dâmaso AR, Agosti F, Zennaro R, de Mello $M T$, et al. Prevalence and risk factors of metabolic syndrome in Brazilian and Italian obese adolescents: a comparison study. Int J Clin Pract. 2008;62:1526-32.

28. Sinaiko A. Obesity, insulin resistance and the metabolic syndrome. J Pediatr (Rio J). 2007;83:3-4.
29. Schwimmer JB, Pardee PE, Lavine JE, Blumkin AK, Cook S. Cardiovascular risk factors and the metabolic syndrome in pediatric nonalcoholic fatty liver disease. Circulation. 2008;118:277-83.

30. Vitola BE, Deivanayagam S, Stein RI, Mohammed BS, Magkos F, Kirk EP, et al. Weight loss reduces fat and improves hepatic and skeletal muscle insulin sensitivity in obese adolescents. Obesity (Silver Spring). 2009;17:1744-8.

31. Saadeh S, Younossi ZM, Remer EM, Gramlich T, Ong JP, Hurley $M$, et al. The utility of radiological imaging in nonalcoholic fatty liver disease. Gastroenterology. 2002;123:745-50.

\author{
Correspondência: \\ Maria Arlete Meil Schimith Escrivão \\ Universidade Federal de São Paulo, Disciplina de Nutrologia, \\ Depto. de Pediatria \\ Rua Loefgreen, 1647 \\ CEP 04040-032 - São Paulo, SP \\ Tel.: (11) 5539.1783 \\ Fax: (11) 5539.1783 \\ E-mail: maria.arlete@uol.com.br, nutsec@yahoo.com.br
}

